\title{
EVALUATION OF PRESENTATION OF SIGNS AND SYMPTOMS OF FEMOROACETABULAR IMPINGEMENT AFTER EPIPHYSIOLYSIS OF THE PROXIMAL FEMUR
}

Fábio Peng Krüger', Paulo Sérgio Gérzon de Britto ${ }^{2}$, Lauro Machado Neto ${ }^{3}$, Carlos Roberto Schwartsmann ${ }^{4}$

\section{ABSTRACT}

Objective: In this study, we sought to evaluate whether there is any relationship between the degree of epiphysiolysis of the proximal femur (EPF) and the presence of femoroacetabular impingement (FAI). Hip range of motion (ROM) was also analyzed in relation to FAI, and the literature on this topic was reviewed. Method: Nineteen cases of EPF in 15 patients who had been treated surgically by means of in situ epiphysiodesis with a cannulated screw were evaluated. The mean follow-up was 27 months. The degree of EPF was analyzed using the epimetaphyseal and neck-epiphyseal angles on lateral-view radiographs, the radiographic signs of FAI on anteroposterior-view radiographs, clinic symptoms and hip ROM. Results: It was found that the degree of EPF (through the epimetaphyseal angle) presented a statistically significant inverse relationship with the presence of FAI over the mean follow-up period of this study. In other words, the patients with symptoms of FAI presented lesser degrees of slippage. This can be explained by the fact that the type of impingement that occurs in cases of EPF (i.e. cam impaction or inclusion) depends on the degree of lippage, and it presents different clinical forms and chronology. The ROM did not present any relationship with FAI. Conclusion: There is a relationship between the degree of slippage and the presence of clinical-radiological FAI after EPF.

Keywords - Epiphysis, slipped/diagnosis; Epiphysis, slipped/epidemiology; Epiphysis, slipped/radiography; Epiphysis, slipped/complications; Hip joint

\section{INTRODUCTION}

Epiphysiolysis of the proximal femur (EPF) is one of the main diseases of the hip in adolescence. Considering its possible short-term complications, chondrolysis and osteonecrosis, careful monitoring is necessary during the initial months of evolution, due to the severity of the sequelae. Such complications have already been covered at length in older studies. However, some studies ${ }^{(1-5)}$ have focused more closely on the possible long-term complications related to femoroacetabular impingement (FAI) and coxarthrosis.

The evolution of EPF to coxarthrosis has long been suggested by many authors in various scientific studies ${ }^{(1-11)}$. More recently, various studies have addressed the precursory relationship between EPF

1 - Resident Doctor of the Orthopedics and Traumatology Service of the Universidade Federal de Ciências da Saúde de Porto Alegre and of the Complexo Hospitalar Santa Casa de Porto Alegre.

2 - Head Doctor of the Department of Children's Orthopedics and Traumatology of the Hospital da Criança Santo Antônio in the Complexo Hospitalar Santa Casa de Porto Alegre.

3 - Assistant Doctor of the Department of Children's Orthopedics and Traumatology of the Hospital da Criança Santo Antônio in the Complexo Hospitalar Santa Casa de Porto Alegre.

4 - Head Doctor of the Orthopedics and Traumatology Service of the Universidade Federal de Ciências da Saúde de Porto Alegre and of the Complexo Hospitalar Santa Casa de Porto Alegre, RS. 
and $\mathrm{FAI}^{(8,10,12-14)}$, including some that have already shown treatment results with satisfactory evolution, through osteochondroplasty, either in isolation or in association with intertrochanteric osteotomy ${ }^{(15-17)}$.

As in any disease, post-EPF FAI presents predictive factors that can help the physician provide a prognosis even before the onset of the symptoms. This is very important in FAI, since by the time symptoms begin, the sequelae may already be severe (chondromalacia, osteoarthritis, bone erosion).

The degree of slippage of EPF can be a predictive factor for the severity of FAI and the chronology of its presentation. In principle, it is believed that the greater the slippage, the higher the chance of presenting FAI, due to the greater metaphyseal gibbosity of the femoral neck impacting the acetabulum. Consequently, the hip range of motion (ROM) can also be a prognostic factor, since the greater the slippage, the greater the restriction of hip movement.

The objective of this study is to ascertain whether a link effectively exists between the degree of EPF slippage and the presentation of post-EPF FAI symptoms. Secondarily, the authors sought to demonstrate the probable relationship between ROM and the presence of FAI, besides reviewing the literature on the subject.

\section{MATERIALS AND METHODS}

Patients with epiphysiolysis of the proximal femur treated surgically at the Hospital da Criança Santo Antônio (Complexo Hospitalar Santa Casa de Porto Alegre) from June 2005 to December 2008 were evaluated retrospectively through the database of the surgical block.

The general symptoms were investigated and linked to the femoroacetabular impingement (hip pain in "C", pain on performing physical activities, pain after sitting for a long time, pain depending on the hip joint position).

In the physical examination, height and body weight were measured to calculate the body mass index (BMI). Flexion, abduction and internal rotation were determined to evaluate the hip ROM, according to the study by Mamisch et $\mathrm{al}^{(12)}$, and according to Tachdjian $^{(18)}$ these are the main movements lost. Internal rotation measurements were obtained with the hip at $90^{\circ}$ of flexion. The FAI test was applied and involved hip flexion, aduction and internal rotation, and the patients were questioned about the presence of pain in the anterior region of the joint ${ }^{(16,19)}$. The Drehmann sign ${ }^{(20)}$ was also investigated.

In the radiographic evaluation, the neck-epiphyseal and epimetaphyseal angles were calculated in lateral-view radiographs of the hip (Lowenstein - frog leg position) to quantify slippage. The neck-epiphyseal angle was measured between a longitudinal line to the center of the neck and another connecting the epiphyseal ends (Figure 1). The epimetaphyseal angle was measured between the latter and the line that connects the two ends of the metaphysis (Figure 2). The following radiological signs of coxarthrosis resulting from FAI were looked for: osteophytes, cysts and/or osteolysis in the femoral head-neck junction; and osteophytes, cysts, labral calcification and/or subchondral sclerosis on the acetabular margin.

For a clinical and radiographic profile to be considered symptomatic of FAI, the patients needed to present, concomitantly, some radiographic alteration, a positive impact test result, and previous painful

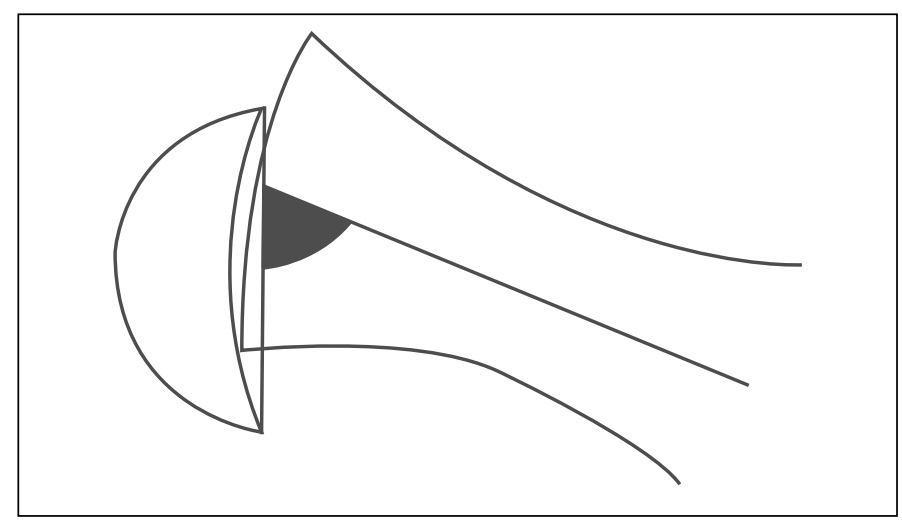

Figure 1 - Neck-epiphyseal angle.

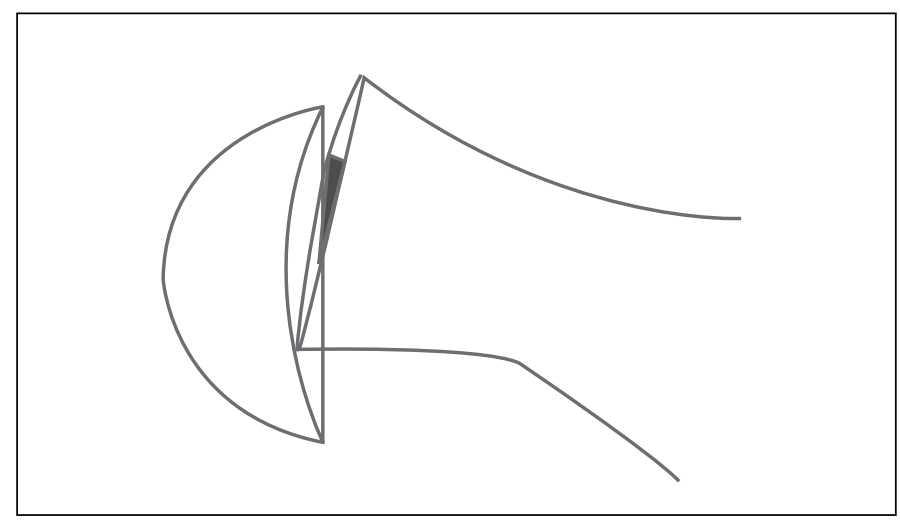

Figure 2 - Epimetaphyseal angle. 
symptoms compatible with FAI. All the patients were assessed clinically and radiologically by the same medical examiner.

\section{RESULTS}

Nineteen cases of epiphysiolysis of the proximal femur were evaluated in a group of 15 patients, consisting of eight men and seven women. The mean age at the onset of initial symptoms was 12 years and five months. The youngest patient was aged 10 years and nine months, and the oldest was 15 years and four months - the only patient aged over 13 . The mean time between surgery and the study interview was two years and three months, with a minimum of nine months and a maximum of 55 months of follow-up time. There were 11 unilateral and four bilateral cases. The right side was affected in eight cases and the left in 11 (Table 1).

All the patients were treated with the same surgical technique - epiphysiodesis of the proximal femur by percutaneous fixation with a $7.0 \mathrm{~mm}$ cannulated screw, on an orthopedic table under fluoroscopic control $^{(21)}$. No maneuvers were performed for reduction of the epiphysiolysis, only positioning on the orthopedic table.
In the clinical evaluation of the 19 cases, four (21\%) presented Drehmann sign, 10 (52\%) presented positive femoroacetabular impingement test results and $12(63 \%)$ presented previous painful symptoms in the hip.

The mean range of motion was 102 degrees of hip flexion, 15.5 degrees of internal rotation and 38 degrees of abduction.

In the radiographic evaluation, the metaphysealepiphyseal angle presented an average of $23^{\circ}$ and the neck-epiphyseal angle $60^{\circ}$ in the lateral view. Thirteen patients $(68 \%)$ exhibited some radiographic sign of impact, and nine of bone remodeling in the femoral neck. The most common coxarthrosis findings related to FAI were acetabular subchondral sclerosis and femoral neck osteolysis. Eight cases fulfilled the three criteria to be considered as having a symptomatic profile of FAI (due to the simultaneous presence of radiographic findings, painful symptoms and positive impact test).

Example of case of a patient with asynchronous (a to o) bilateral EPF, measurement of the epimetaphyseal and neck-epiphyseal angles (b, h), evolution demonstrating substantial remodeling (d, f; j, m) (Figure 3).

The comparison between the degree of slippage, through the epimetaphyseal angle, and the sympto-

Table 1 - Particulars of the patients analyzed.

\begin{tabular}{|c|c|c|c|c|c|c|c|c|c|}
\hline Patient & Side & Gender & Age $(y+m)$ & Follow-up (m) & Weight (kg) & Height (m) & BMI $\left(\mathrm{kg} / \mathrm{m}^{2}\right)$ & \begin{tabular}{|} 
Neck- \\
epiphyseal \\
angle
\end{tabular} & $\begin{array}{c}\text { Epimetaphyseal } \\
\text { angle }\end{array}$ \\
\hline 1 & $r$ & $f$ & 13 & 27 & 52 & 1.56 & 21.37 & 58 & 8 \\
\hline 2 & $r$ & $f$ & $11+7$ & 9 & 56 & 1.52 & 24.24 & 70 & 10 \\
\hline 3 & $r$ & $f$ & $12+6$ & 24 & 74 & 1.65 & 27.18 & 70 & 10 \\
\hline 4 & I & $\mathrm{m}$ & $13+1$ & 31 & 74 & 1.6 & 28.91 & 70 & 10 \\
\hline 5 & $r$ & $f$ & $11+4$ & 10 & 54 & 1.59 & 21.36 & 18 & 12 \\
\hline 6 & $r$ & $\mathrm{~m}$ & $10+9$ & 14 & 71 & 1.61 & 27.39 & 72 & 12 \\
\hline 7 & 1 & $f$ & $12+4$ & 35 & 52 & 1.56 & 21.37 & 65 & 12 \\
\hline 8 & 1 & $f$ & $13+3$ & 15 & 74 & 1.65 & 27.18 & 66 & 12 \\
\hline 9 & I & $m$ & $13+5$ & 10 & 42 & 1.58 & 16.82 & 62 & 16 \\
\hline 10 & $r$ & $\mathrm{~m}$ & 12 & 42 & 74 & 1.6 & 28.91 & 66 & 18 \\
\hline 11 & $r$ & $\mathrm{~m}$ & $12+11$ & 44 & 70 & 1.64 & 26.03 & 98 & 18 \\
\hline 12 & I & $m$ & $13+11$ & 32 & 70 & 1.64 & 26.03 & 100 & 18 \\
\hline 13 & 1 & $\mathrm{~m}$ & $12+11$ & 47 & 106 & 1.84 & 31.31 & 54 & 20 \\
\hline 14 & $r$ & $\mathrm{~m}$ & $10+9$ & 36 & 77 & 1.76 & 24.86 & 24 & 32 \\
\hline 15 & 1 & $\mathrm{~m}$ & 15 & 19 & 79 & 1.71 & 27.02 & 50 & 36 \\
\hline 16 & $I$ & $f$ & 11 & 17 & 73 & 1.55 & 30.39 & 56 & 40 \\
\hline 17 & 1 & $\mathrm{~m}$ & 12 & 30 & 59 & 1.68 & 20.90 & 74 & 46 \\
\hline 18 & 1 & $\mathrm{~m}$ & 12 & 9 & 72 & 1.52 & 31.16 & 44 & 46 \\
\hline 19 & I & $f$ & 11 & 44 & 85 & 1.6 & 33.20 & 32 & 60 \\
\hline
\end{tabular}



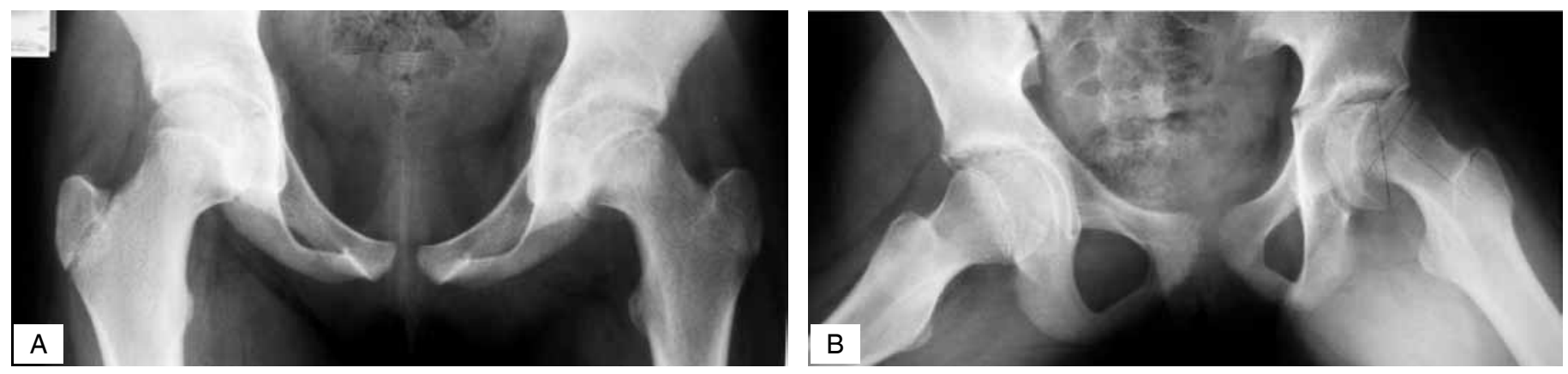

Figure $3-a, b)$ Onset of symptoms in L hip in Oct/06.
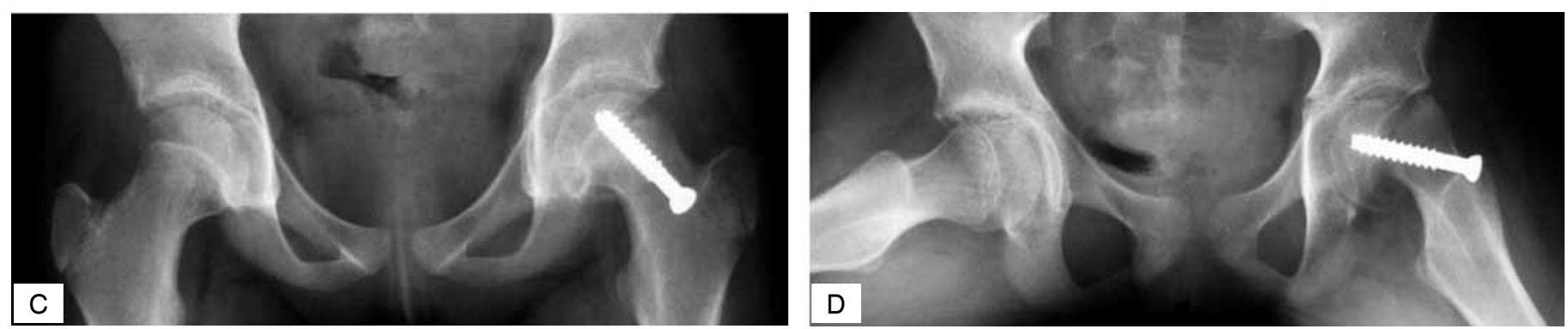

Figure $3-c, d$ ) Postoperative L hip (one month) in Nov/06.
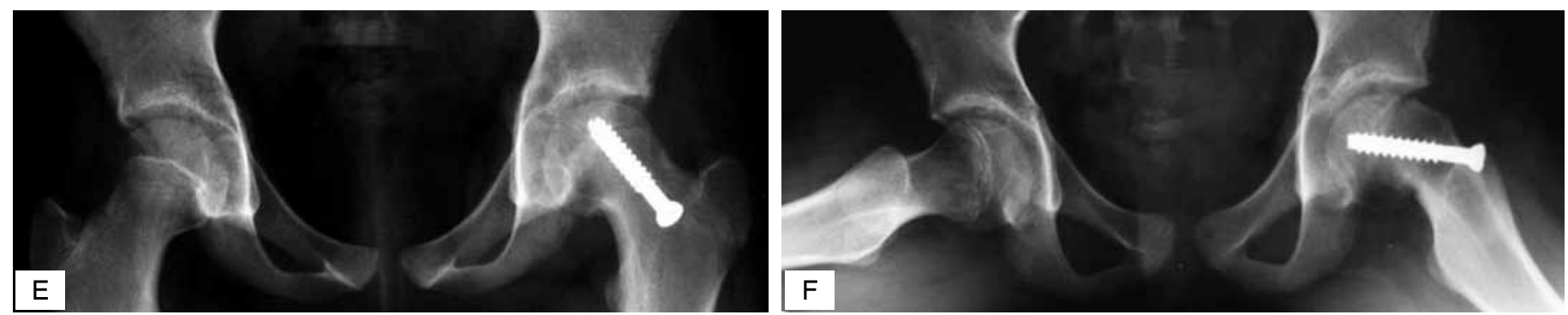

Figure 3 - e, f) Postoperative L hip (five months) in Mar/07.
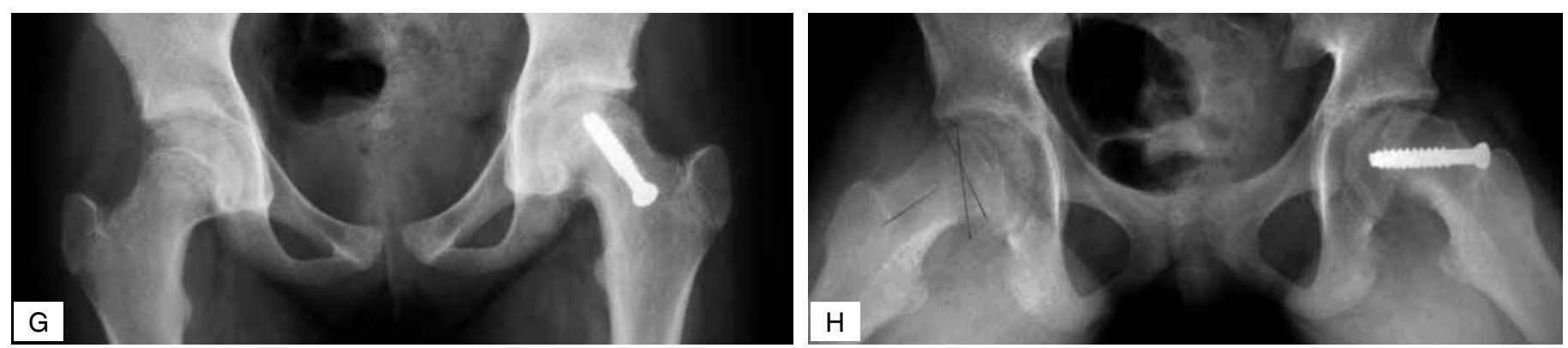

Figure $3-\mathrm{g}$, h) Onset of symptoms in R hip and postoperative L hip (seven months) in May/07.
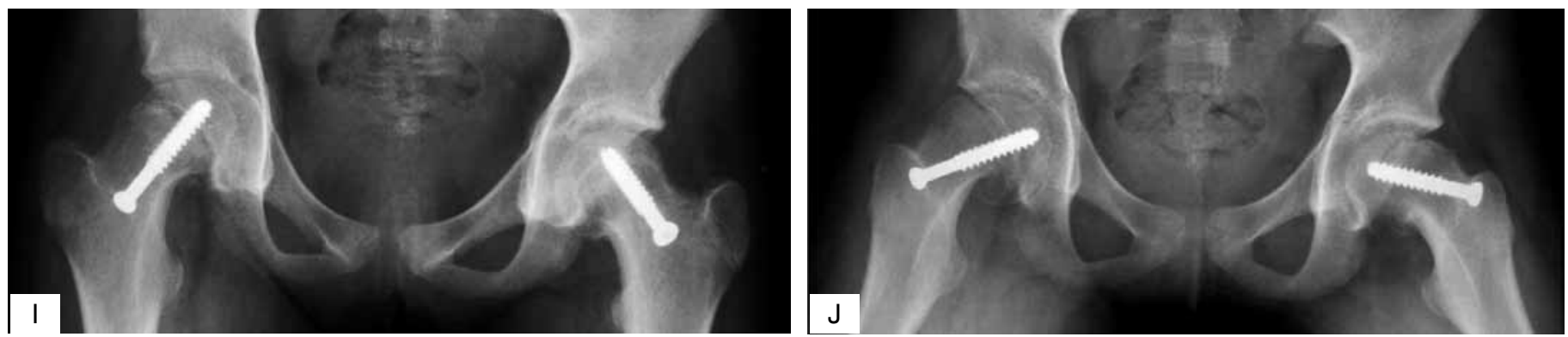

Figure 3 - i, j) Postoperative R (one month) and L hip (nine months) in Jul/07. 

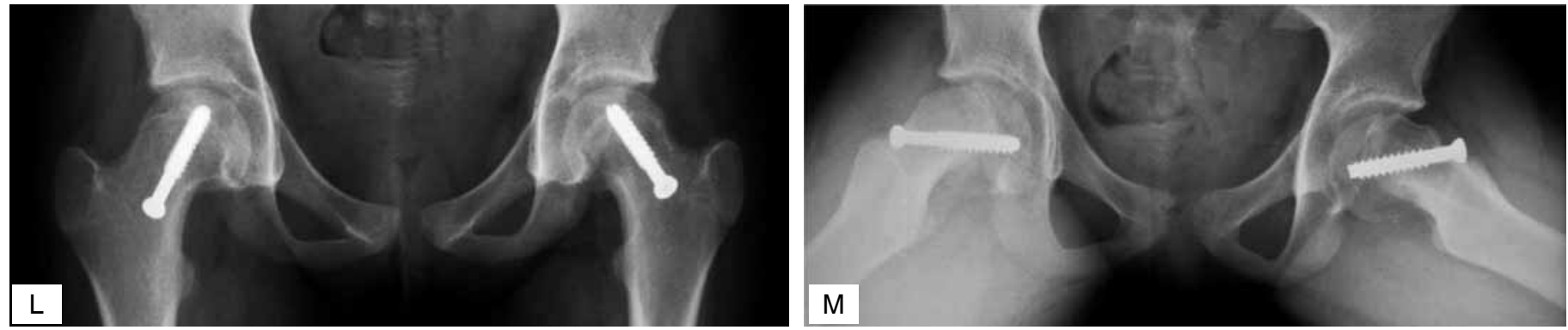

Figure 3 - I, m) Postoperative L hip (two months) and R hip (10 months) in Aug/07.
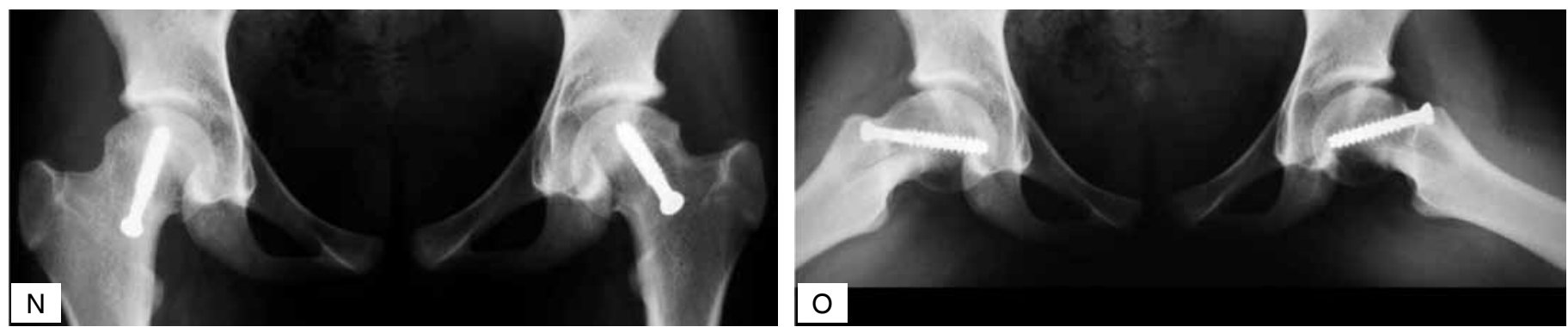

Figure 3 - n, o) Postoperative R (22 months) and L hip (30 months) in Apr/09.

matic profile of FAI showed evidence of a statistically significant inverse relationship $(p=0.045$ in the Kruskal-Wallis test; ANOVA, Parametric Test for Inequality of Population Means $p=0.0534)$. The patients with a symptomatic profile of FAI at the time of the interview presented a smaller epimetaphyseal angle than those without this symptomatic profile. However, when the neck-epiphyseal angle was used to rate the slippage, there was no statistically significant relationship between these variables.

ROM was not related to the presence of FAI, calculating each movement (flexion, internal rotation and abduction) separately (Table 2).

Table 2 - Range of movement according to the presence of a symptomatic profile of FAI.

\begin{tabular}{l|c|c}
\hline & FAl+ & FAl- \\
\hline Flexion & 108.75 & 116.6 \\
\hline Abduction & 43.1 & 33 \\
\hline Internal rotation & 18.1 & 14.1 \\
\hline
\end{tabular}

\section{DISCUSSION}

The sample was homogeneous, with uniformity in terms of age and gender of the population studied. The number of patients of the sample group was smaller than expected, hindering the calculations and reducing their statistical power.
All the patients of this study had more than six months of postoperative evolution, the period with the greatest gain in $\mathrm{ROM}^{(19)}$. Although there is evidence that FAI can lead to changes in the ROM, in this study there was no statistical difference in the relationship between ROM and the angles (neck-epiphyseal or epimetaphyseal). However, Mamisch et $\mathrm{al}^{(12)}$ conclude, in their recent study, that the quantity of slippage and the morphology of the femoral metaphyseal prominence influence the ROM, and these can be considered important variables in future studies ${ }^{(8)}$.

Paradoxically, an unexpected inverse relationship was found between the quantity of slippage and the presence of FAI symptomatology, when considering the epimetaphyseal angle. In other words, the smaller the angle, the greater the chance of presenting the signs and symptoms of FAI investigated in this study. We had expected the exact opposite: the greater the slippage, the greater the friction between the acetabular margin and the prominent femoral neck, causing more FAI symptoms. Yet this relationship does not appear to be direct.

The type of FAI that occurs after EPF is cam, which is based on a prominence of the anterolateral region of the femoral neck and can have several origins. Depending on the size of this defect, this prominence can penetrate the acetabulum and cause direct abrasions to the cartilage, resulting in the cam inclusion sub- 
type; or it can impact the acetabular margin, without managing to penetrate the joint, causing immediate labral and indirect cartilaginous lesions, resulting in the cam impaction subtype. Each type presents signs and symptoms at different times during the evolution of the disease ${ }^{(10)}$.

Moreover, according to $\mathrm{Rab}^{(10)}$, the determination of which cam impact subtype is expected to occur (inclusion or impaction) will depend on the degree of slippage. Generally, a slight degree causes the inclusion subtype and larger degrees cause the impaction subtype. Each cam subtype has individual forms of presentation. The inclusion subtype generates symptoms sooner and produces hip osteoarthritis earlier, due to the direct lesion of the cartilage of the weight-bearing area (acetabular dome); in this scenario, the patient does not exhibit major limitations of the ROM, since the defect "fits" inside the joint. The impaction subtype generates symptoms and osteoarthritis later, since the initial direct mechanical lesion occurs between metaphyseal prominence of the femoral neck and the acetabular margin, composed of the fibrocartilaginous labrum, hipbone and articular cartilage adjacent to the labrum (in other words, the extreme outer part of the acetabulum). Thus, the articular cartilage of the hip weight-bearing area is initially preserved, and is only affected with the consequent progression of the disease $\mathrm{e}^{(10)}$. In the last model, the ROM is seriously limited, as the defect does not manage to enter the articular space.

Based on these facts, it can be assumed that the onset and the form of presentation of post-EPF FAI symptoms will depend on the type of impact (caminclusion or cam-impaction) and not directly on the degree of slippage.

In addition to the previous explanation, there are other factors that could influence the presentation of FAI symptoms in EPF. Patients compensate the deformity in variable and individual ways, adopting adaptive positions (e.g.: increasing external rotation and decreasing hip flexion) and refraining from physical activities of their own accord, always seeking to avoid the phenomenon of $\mathrm{FAI}^{(10)}$.

The two cam impact subtypes should be viewed dynamically, as they can change with time and function (e.g.: through remodeling of the femoral neck or gait alterations). Also, for this reason, some patients appear to tolerate more slippage than others, mainly through the bone remodeling that occurs more in younger patients.

The neck-epiphyseal angle did not show any relation to the presence of FAI. One of the possible reasons for this is the difficulty in obtaining uniformity in frog leg position radiographs, and it is sometimes complicated to draw the median line of the femoral neck to measure the angle. The other reason is the small number of cases in our series.

In Table 2, the patients of the FAI+ group represent the group of symptomatic individuals (positive triad: anamnesis, physical examination, and radiograph) at the time of the study, with probable cam impact of the inclusion subtype, in which there is greater range of motion than the other group, with probable impaction. The abduction and internal rotation corroborated this hypothesis, and were greater in the FAI+ group; yet flexion was greater in the FAI group.

The measurements of the epimetaphyseal and neckepiphyseal angles are sometimes hard to gauge in the authors' opinion, due to the technical difficulty in some exams. Nonetheless, the literature contains studies that qualify this as a valid form of measurement, with good rates of inter- and intra-observer reproducibility ${ }^{(22)}$.

The same results were not found in studies with similar investigation goals. DeLullo et $\mathrm{al}^{(23)}$ and Dodds et $\mathrm{al}^{(24)}$ concluded that the severity of slippage did not influence the incidence of appearance of FAI symptoms.

The authors consider that the FAI diagnosis can be made for the patient as soon as the EPF diagnosis has been confirmed; considering that there will be an increase in angle $\alpha$ and decrease in the cervico-cephalic offset automatically when the femoral neck slides in the anterosuperior direction. In other words, every patient with EPF has FAI of the cam type, which may be symptomatic or asymptomatic.

The questions that remain are: which subtype the patient will develop (inclusion or impaction), when complaints will begin, and the magnitude of sequelae. Patients with a smaller degree of slippage in EPF appear to have early signs and symptoms of FAI, probably because they present the inclusion subtype of the cam impact type, which does little to restrict hip ROM, but causes cartilaginous alterations to occur sooner. On the other hand, patients with a greater degree 
of slippage appear to present late signs and symptoms of FAI, as they probably present the impaction subtype of the cam impact type, which classically causes a significant limitation of the ROM, initial labral lesions, and long-term cartilaginous lesions.

Many studies are appearing in the literature on the subject of coxarthrosis and post-EPF FAI. Abraham et $\mathrm{al}^{(13)}$ distinguished between idiopathic coxarthrosis and coxarthrosis secondary to EPF, considering that the latter presents loss of the cervico-cephalic femoral offset, presence of FAI and peripheral acetabular cartilage lesion adjacent to the lateral superior femoral neck, while the idiopathic type does not present these findings. They also demonstrated that patients with post-EPF coxarthrosis are submitted to total hip arthroplasty 11 years earlier than patients with idiopathic coxarthrosis.

Thus we are already far from the old idea that included coxarthrosis due to epiphysiolysis within the idiopathic diseases. This study serves as an example for wide studies, seeking to prove possible relationships between the presentation of post-EPF FAI, the ROM and the degree of slippage.

\section{CONCLUSION}

Patients with symptomatic post-EPF FAI presented smaller degrees of slippage. The ROM was not related to the degree of slippage.

\section{REFERÊNCIAS}

1. Goodman DA, Feighan JE, Smith AD, Latimer B, Buly RL, Cooperman DR Subclinical slipped capital femoral epiphysis. Relationship to osteoarthrosis of the hip. J Bone Joint Surg Am. 1997;79(10):1489-97.

2. Murray RO. The aetiology of primary osteoarthritis of the hip. Br J Radiol. 1965;38(455):810-24.

3. Stulberg SD, Cordell LD, Harris WH, Ramsey PL, MacEwen GD. Unrecognized childhood hip disease: a major cause of idiopathic osteoarthritis of the hip. In: Amstutz HC, editors. The Hip: Proceedings of the Third Open Scientific Meeting of the Hip Society. St Louis: Mosby; 1975;212-28.

4. Harris WH. Etiology of osteoarthritis of the hip. Clin Orthop Relat Res. 1986;(213):20-33.

5. Aronson J. Osteoarthritis of the young adult hip: etiology and treatment. Instr Course Lect. 1986;35:119-28.

6. Boyer DW, Mickelson MR, Ponseti IV. Slipped capital femoral epiphysis. Long-term follow-up study of one hundred and twenty-one patients. J Bone Joint Surg Am. 1981;63(1):85-95.

7. Carney BT, Weinstein SL, Noble J. Long-term follow-up of slipped capital femoral epiphysis. J Bone Joint Surg Am. 1991;73(5):667-74.

8. Leunig M, Casillas MM, Hamlet M, Hersche O, Nötzli H, Slongo T, Ganz R. Slipped capital femoral epiphysis: early mechanical damage to the acetabular cartilage by a prominent femoral metaphysis. Acta Orthop Scand. 2000;71(4):370-5.

9. Richolt JA, Teschner M, Everett PC, Millis MB, Kikinis R. Impingement simulation of the hip in SCFE using 3D models. Comput Aided Surg. 1999;4(3):144-51.

10. Rab GT. The geometry of slipped capital femoral epiphysis: implications for movement, impingement, and corrective osteotomy. J Pediatr Orthop. 1999;19(4):419-24.

11. Wilson PD, Jacobs B, Schecter L. Slipped capital femoral epiphysis: an endresult study. J Bone Joint Surg Am. 1965;47:1128-45.

12. Mamisch TC, Kim YJ, Richolt JA, Millis MB, Kordelle J. Femoral morphology due to impingement influences the range of motion in slipped capital femoral epiphysis. Clin Orthop Relat Res. 2009;467(3):692-8.

13. Abraham E, Gonzalez MH, Pratap S, Amirouche F, Atluri P, Simon P. Clinical im- plications of anatomical wear characteristics in slipped capital femoral epiphysis and primary osteoarthritis. J Pediatr Orthop. 2007;27(7):788-95.

14. Nötzli HP, Wyss TF, Stoecklin CH, Schmid MR, Treiber K, Hodler J. The contour of the femoral head-neck junction as a predictor for the risk of anterior impingement. J Bone Joint Surg Br. 2002;84(4):556-60.

15. Spencer S, Millis MB, Kim YJ. Early results of treatment of hip impingement syndrome in slipped capital femoral epiphysis and pistol grip deformity of the femoral head-neck junction using the surgical dislocation technique. J Pediatr Orthop. 2006;26(3):281-5.

16. Crestani MV, Telöken MA, Gusmão PDF. Impacto femoroacetabular: uma das condições precursoras da osteoartrose do quadril. Rev Bras Ortop. 2006;41(8):285-93.

17. Schai PA, Exner GU. Corrective Imhäuser intertrochanteric osteotomy. Oper Orthop Traumatol. 2007;19(4):368-88.

18. Tachdjian MO. Clinical pediatric orthopedics: the art of diagnosis and principles of management. Stamford, Connecticut: Appleton and Lange; 1997.

19. Sink EL, MD, Gralla J, Ryba A, Dayton M. Clinical Presentation of Femoroacetabular Impingement in Adolescents. J Pediatr Orthop. 2008; 28(8):806-11.

20. Drehmann F. Drehmann's sign. A clinical examination method in epiphysiolysis (slipping of the upper femoral epiphysis). Description of signs, aetiopathogenetic considerations, clinical experience. Z Orthop Ihre Grenzgeb. 1979;117(3):333-44.

21. Aronson DD, Carlson WE. Slipped capital femoral epiphysis. A prospective study of fixation with a single screw. J Bone Joint Surg Am. 1992;74(6):810-9.

22. Siegel DB, Kasser JR, Sponseller P, Gelberman RH. Slipped capital femoral epiphysis. A quantitative analysis of motion, gait, and femoral remodeling after in situ fixation. J Bone Joint Surg Am. 1991;73(5):659-66.

23. DeLullo JA, Thomas E, Cooney TE, McConnell SJ, Sanders JO. Femoral remodeling may influence patient outcomes in slipped capital femoral epiphysis. Clin Orthop Related Res. 2007;457:163-70.

24. Dodds MK; McCormack D; Mulhall KJ. Femoroacetabular impingement after slipped capital femoral epiphysis: does slip severity predict clinical symptoms? J Pediatr Orthop. 2009;29(6):535-9. 